\title{
Partial Disjoint Path for Multi-layer Protection in GMPLS Networks
}

\author{
Anna Urra, Student Member, IEEE, Eusebi Calle, Jose L. Marzo, Member, IEEE \\ Institute of Informatics and Applications (IIiA), University of Girona, 17071 Girona, Spain \\ E-mail: \{aurra, eusebi, marzo\}@eia.udg.es
}

\begin{abstract}
In this paper, different recovery methods applied at different network layers and time scales are used in order to enhance the network reliability. Each layer deploys its own fault management methods. However, current recovery methods are applied to only a specific layer. New protection schemes, based on the proposed Partial Disjoint Path algorithm, are defined in order to avoid protection duplications in a multi-layer scenario. The new protection schemes also encompass shared segment backup computation and shared risk link group identification. A complete set of experiments proves the efficiency of the proposed methods in relation with previous ones, in terms of resources used to protect the network, the failure recovery time and the request rejection ratio.
\end{abstract}

Index Terms - quality of service, reliability, shared segment protection, GMPLS.

\section{INTRODUCTION}

Ensuring a particular level of reliability is necessary when the required transmissions are critical or prioritized. In order to enhance the network reliability, different recovery methods applied at different network layers and time scales are used. The use of optical network technology in core network combined with Multi-Protocol Label Switching (MPLS) for offering traffic-engineering capabilities has been selected as a suitable choice in many current Internet Service Provider (ISP) networks. The integration of MPLS and optical network is facilitated by the development of Generalized MPLS (GMPLS) [1]. Both optical and MPLS layers have deployed their own fault management methods [2]. However, some of the current recovery methods, such as $[3,4,5]$, are only applied to a specific layer. On the other hand, fault management can be offered at link or node level. However, node failures can be seen as the failure of all the links within this node. Thereby, in this paper we take into account single link failures.

This paper focuses on enhancing the online MPLS routing when protection duplications are avoided. One example is shown in Fig. 1. The working path between the node pair $(3,2)$ does not need to establish a backup path because the logical link 2-3 at MPLS layer, i.e. the lightpath $\mathrm{L}_{1}$ (3-1-2) at the optical layer, is already protected by the backup lightpath $\mathrm{BL}_{1}$ (3-4-2). Thus, the multi-layer fault

\footnotetext{
* This work was supported by the COST 293 and the Spanish Research Council (CICYT) under contract TIC2003-05567. The work of A. Urra was also supported by the Ministry Universities, Research and Information Society (DURSI) and European Social Funds.
}

management is simplified and the resource consumption is reduced. In order to deploy this idea, a necessary new definition of link-disjoint path using Shared Risk Link Group (SRLG) [6] adapted to multi-layer protection is introduced.

This paper is organized as follows. In section II, the existing routing algorithms are analyzed. Section III proposes novel protection schemes to improve network reliability and reduce the impact of the link failure, minimizing the failure recovery time. The simulation scenarios and performance results are presented in section IV. Section V concludes the paper.

\section{LITERATURE SURVEY}

Network protection is usually based on the establishment of a link-disjoint path pairs: the working path (WP) and the backup path (BP). When a link failure occurs the WPs, which are affected by the link failure, switch over the traffic to their respective BP. One example of disjoint path-pairs routing algorithm was introduced by Suurballe [7]. Although Suurballe's algorithm is optimal and has polynomial computational complexity, it is only oriented to dedicated protection. Since resources are not shared in dedicated protection, there is poor resource utilization. Shared protection outperforms dedicated protection in terms of resource consumption but, in order to provide efficient resource consumption, the WP links must be known before BP computation [5]. Therefore, a two-step routing algorithm is necessary when shared protection is used.

In this section, some existent routing algorithm proposals are analyzed.

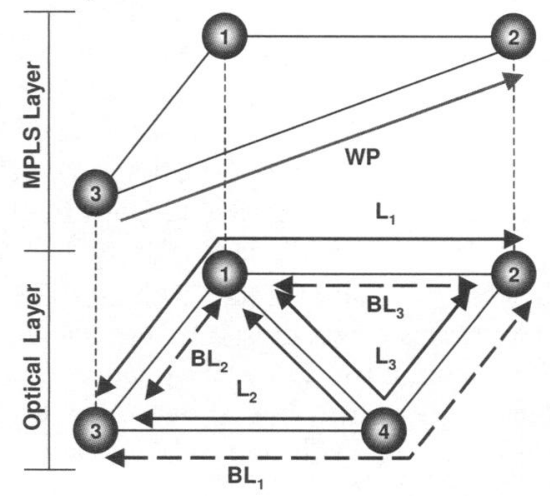

L: Lightpath BL: Backup Lightpath WP: Working Path

Figure 1 - Multi-layer protection. 


\section{A. QoS Routing Algorithms}

Different QoS routing criterion are found in the literature in order to compute the working path (WP). For instance, the Min-Hop Algorithm (MHA) selects a feasible path with the least number of hops (links).

Traditional QoS routing algorithms, such as the wellknown Widest-Shortest Path (WSP) [8] routing algorithm, use two different objective functions to optimize network performance, where by the shortest path is selected for minimizing cost and the least loaded path is selected for load balancing.

Another recent proposal of WP computation was presented in [9] where, although the multi-layer is taken into account for WP computation, fault management methods are not considered.

\section{B. QoS with Protection Routing Algorithms}

A crucial aspect in the development of a fault management system is the selection of backup paths (BPs). Although routing algorithms reviewed in the above mentioned section (MHA, WSP) can be used to compute the BP, they do not include any objective to actually improve the protection level such as the maximization of the shared bandwidth or the minimization of the fault recovery time.

Shared protection schemes are developed depending on the available network information. A proposal to compute shared BP based on a Partial Information Routing (PIR) was introduced in [10]. In [11] a proposal of Full Information Routing (FIR) was presented. The main idea of FIR is based on assigning weight to each link based on the maximum bandwidth needed if any of the links of the protected path fail and if any of the network links fail. FIR performs better compared to the previous proposals only when the required routing information is available. This drawback can be solved using signaling techniques.

Marzo [12] presents local, segment and global MPLS protection methods. Local and global protection can be shown as particular cases of the segment protection based on the notification time, i.e. the time required to notify the fault from the node detecting the failure and the node responsible for the switchover. Notification time is probably one of the main aspects to be reduced in order to minimize the fault recovery time and offer faster protection [5]. In the case of local protection, the notification time is 0 , since the node that detects the failure is also responsible for the traffic switchover. In the global case, the node that detects the failure must send a Fault Indication Signal (FIS) to the ingress node (source) reporting large failure recovery time when the failure occurs close to the destination node. Although local protection has the lowest recovery time, protecting the whole network with local $\mathrm{BP}$ results in highest resource consumption. A trade-off exists between the resource consumption and the failure recovery time minimization. Therefore, the use of segment protection is proposed.

Our proposed algorithms compute the BP using Shared Segment Protection (SSP). We present heuristic approaches because of the NP-completeness of the SSP problem [13].

\section{PROBLEM STATEMENT}

In this section the basis of our novel proposed algorithms are discussed. The network scenario and the problem formulation are also described.

\section{A. Fundamentals of Partial Disjoint Path}

There are certain trade-off issues between the resource consumption and signaling overheads depending on the layer used [15] for fault management.

In this paper, a partially protected optical layer is proposed. Therefore, no extra resource is necessary in the MPLS layer against failure of protected links in the optical layer. Once the working path (WP) is known, the backup path (BP) is computed. As a novelty, the backup path is proposed to be a Partial Disjoint Path (PDP) since it may overlap the nodes of the WP and the links of the WP that are already protected at the optical layer. The definition of the proposed PDP algorithm is presented in Section III.C. When the PDP overlaps with the WP, more than one backup path, i.e. segment backup paths (SBP), are established. Hence, when a PDP is computed, the optical protected links may: a) not belong to the protected segment
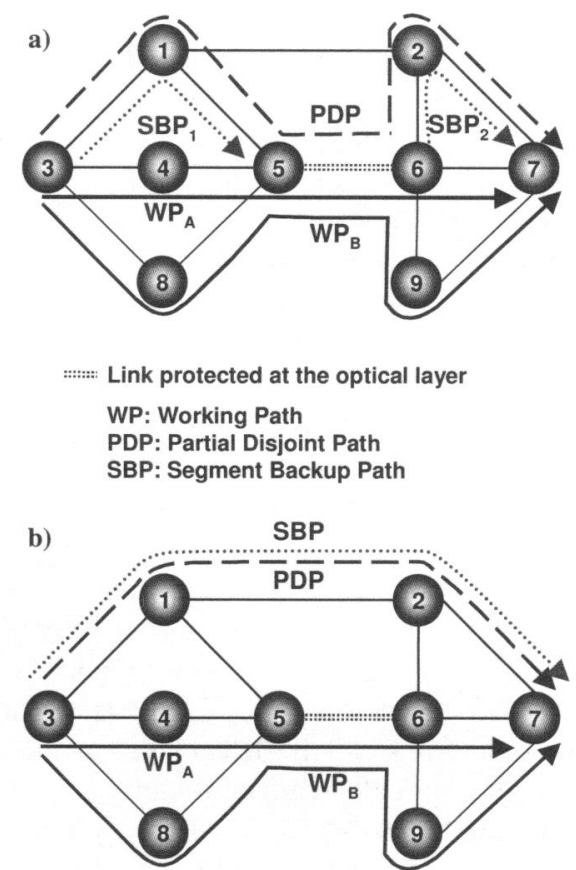

Figure 2 - MPLS protection when the Partial Disjoint Path a) overlaps protected links at the optical layer b) does not overlap the protected links at the optical layer. 
path, b) belong to the protected segment path. Both cases are shown in Fig. 2a and $2 \mathrm{~b}$ respectively. In Fig. 2, two WPs are established sharing the link 5-6 that is protected at the optical layer. The same PDP is used to protect both the WPs. In the first case a), the computed PDP overlaps $W_{A}$ and $\mathrm{WP}_{\mathrm{B}}$. This means that two segment backup paths $\left(\mathrm{SBP}_{1}\right.$ and $\mathrm{SBP}_{2}$ ) are established between the protected segment paths 3-4-5 and 6-7 since the link 5-6 is already protected. Moreover, the SBP bandwidth is shared in both cases (Fig. $2 a$ and $2 b$ ) since the shared link 5-6 does not need to be protected at the MPLS layer. This is not possible if definition of link-disjoint path based on Shared Risk Link Group (SRLG) is considered [6]. Two data paths are linkdisjoint if no two links on the two paths belong to the same SRLG. As shown in Fig. $2 b$, both the $W_{\mathrm{A}}$ and the $\mathrm{WP}_{\mathrm{B}}$ belong to the same SRLG since they are sharing link 5-6, thus backup path capacity is not sharable. However, this link is already protected at the optical layer and, consequently, the SBP defined at the MPLS layer is not activated against the failure of link 5-6. Therefore, in the multi-layer scenario considered in this paper, two data paths are link-disjointed if the links that are unprotected do not belong to the same SRLG.

\section{B. Network scenario}

Let $G=(V, E)$ describe the given network, where $V$ is the set of network nodes, and $E$ is the set of network links. Each link $(i, j) \in E$ has an associated $L_{i j}$ physical length; $R_{i j}$ residual bandwidth; $D_{i j}$ total bandwidth dedicated to working path; $S_{i j}^{u v}$ total bandwidth reserved to protect link $(u, v)$; and $T_{i j}$ the total shared bandwidth allocated in link $(i$, $j)$. Note that $S_{i j}^{u v}$ is equal to 0 when the link $(u, v)$ is protected at the optical layer and $T_{i j}=\max _{(u, v) \in E}\left(S_{i j}^{u v}\right)$.

Assuming that there is a set of distinguished node pairs $P$, which may be thought of as a set of potential ingressegress node pairs, all connection set-up requests occur between these pairs. We denote a generic element of this set by $(s, d)$.

The set up request is defined by $(s, d, b)$ where $b$ specifies the amount of bandwidth required for this request. For each set up request, a working path (WP) has to be setup and a backup path (BP) must also be set up if the LSP has at least one link to protect. If there is not a sufficient bandwidth in the network for either the working path or the backup path for the current request, the request is rejected. Here, we neither assume any knowledge about future requests nor any statistical traffic profile.

\section{Partial Disjoint Path Algorithm}

A Partial Disjoint Path (PDP) is computed in order to identify the segment backup paths necessary to protect the working path (see section III.A). First, a weight $w_{i j}$ is assigned on each link according to Equation 1:

$$
w_{i j}= \begin{cases}0 & \text { if }(i, j) \in W P \text { and } p_{i j}=1 \\ c_{i j} & \text { if }(i, j) \notin W P \text { and } p_{i j}=0 \text { and } R_{i j}+T_{i j}-A \geq b \\ \infty & \text { otherwise }\end{cases}
$$

Where $A$ is the maximum capacity necessary if one of the unprotected WP links fails; $c_{i j}$ is the cost assigned to link $(i$, $j$ ) according to FIR algorithm [11]; and $p_{i j}$ contains 1 if link $(i, j)$ is protected at the optical layer, 0 otherwise. Once the weight is assigned the PDP is computed. This is done by a variation of Dijkstra's algorithm called PartialDisjointPath.

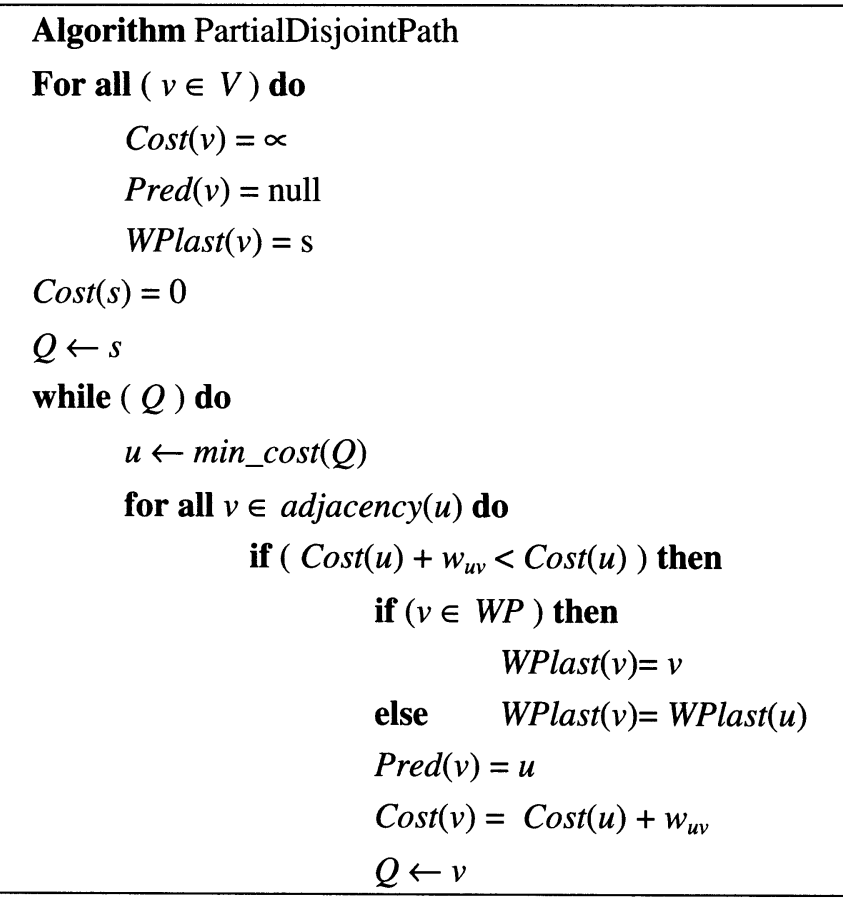

In this algorithm $\operatorname{Cost}(v)$ is a vector which contains the path cost from $s$ to $v$; Pred $(v)$ contains the $v$ 's predecessor node; and WPlast $(v)$ contains the last WP node visited before treating node $v . Q$ represents the list of adjacent vertices which were not yet visited. Function $\min \_\operatorname{cost}(Q)$ returns the element $u \in Q$ with the lowest $\operatorname{Cost}(u)$; and adjacent $(u)$ represents the adjacency list of vertex $u$.

Once the PDP is computed, the BP links are identified. Only the links of the PDP, which do not belong to the WP, are the backup links. Other links are considered unprotected at MPLS layer since they are protected at the optical layer. The reserved bandwidth will depend on the amount of bandwidth that may be shared in each backup link and the links that are protected at MPLS layer.

\section{Online QoS Restorable Routing Schemes}

We assume that the WP is selected as the WSP since the purpose of this paper is to select the restoration path in order to minimize the total reserved restoration resources 
over all network links in the MPLS layer, taking into account the WDM layer protection.

We propose two routing schemes based on the proposed PDP routing algorithm as follows:

Partial Disjoint Path with Full Information (PDPFI). This algorithm uses the PDP to compute the BP.

Partial Disjoint Path with Partial Information (PDPPI). This algorithm uses a variation of the PDP to compute the BP. In PDPPI, the cost $c_{i j}$ given in Equation 1 is assigned according to PIR algorithm.

In order to compare our proposals, the next two algorithms without multi-layer differentiation are also considered:

Full Disjoint Path with Full Information (FDPFI). This algorithm has the objective of minimizing the resource consumption used in the backup path. Therefore, FIR [11] is used to compute the backup path.

Widest Shortest Path (WSP). This algorithm uses the WSP to compute the BP.

\section{PERFORMANCE EVALUATION}

\section{A. Network Topology}

For this set of experiments the KL topology shown in Fig. 3 was used. The capacity of the links was 1200 and 4800 units, representing OC-12 and OC-48 rates, respectively. Each link was bi-directional i.e., it acted like two unidirectional links of that capacity. There were 15 nodes and 28 links.

Requests arrived according to a Poisson process with an average rate $\lambda$, and exponentially distributed holding times with a mean value of $1 / \mu$. For this set of experiments, $\lambda / \mu$ was 150 . Ten independent trials were performed over a window of 10,000 Label Switched Paths (LSPs) set-up requests.

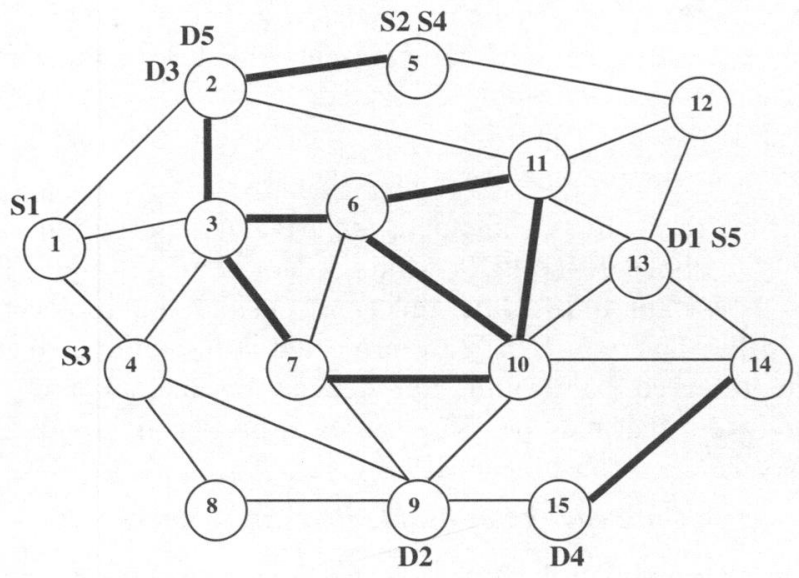

Figure 3 - KL topology.
In order to compute the failure notification time, a link length was assigned randomly between 200 and 1000 miles for each network link.

\section{B. Figures of Merit}

To evaluate the algorithm performances, three figures of merit were used in the experiments viz. 1) the request rejection ratio; 2) the restoration overbuild, percentage of bandwidth used as a BP with respect to the bandwidth used as a WP; and 3) the failure notification distance, analysis of failure notification time in terms of notification distance.

In order to evaluate different network scenarios in terms of protection requirements, the number of links that should be protected in the network was used.

\section{Simulation Results}

First of all, the failure recovery time is analyzed in terms of failure notification distance and protection requirements. In the 14 links to protect case shown in Fig. 4a, the algorithms that use the proposed protection schemes (PDPPI and PDPFI) accumulate more LSPs with the notification distance between 0 and 400 miles because of the application of Partial Disjoint Path algorithm. Since the
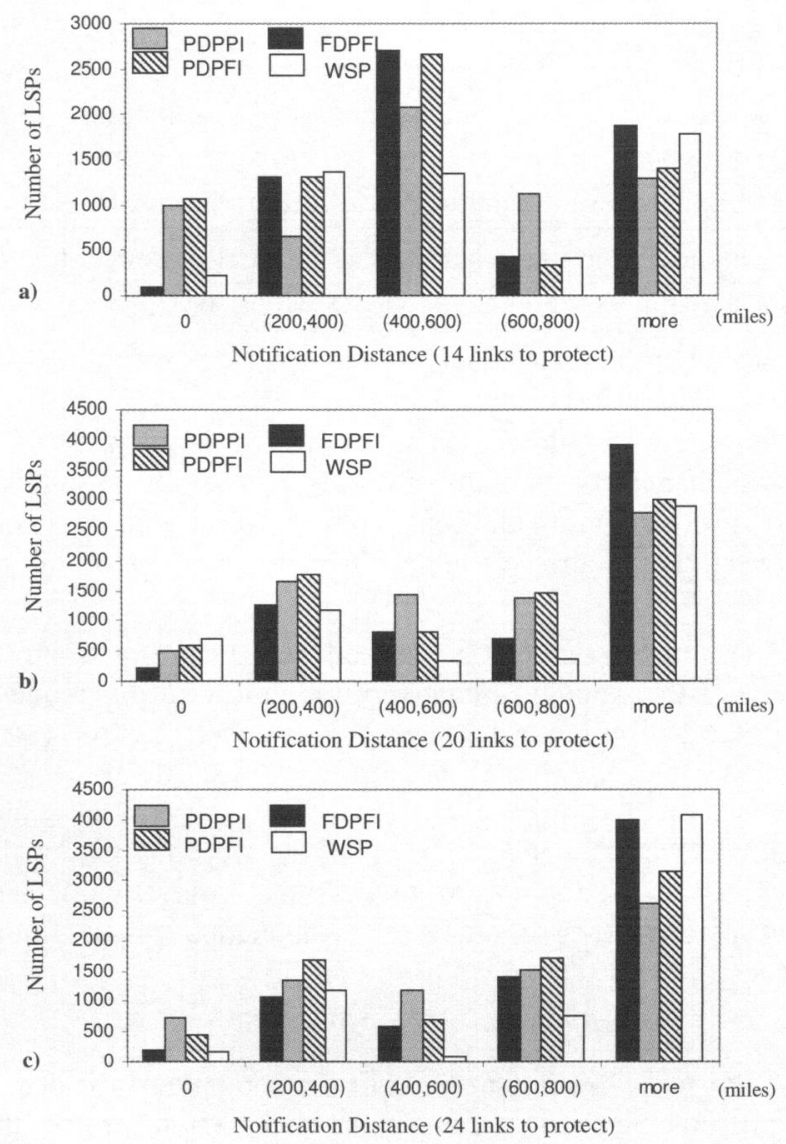

Figure 4 - Failure notification distance for a network with a) 14 links to protect b) 24 links to protect c) 24 links to protect. 

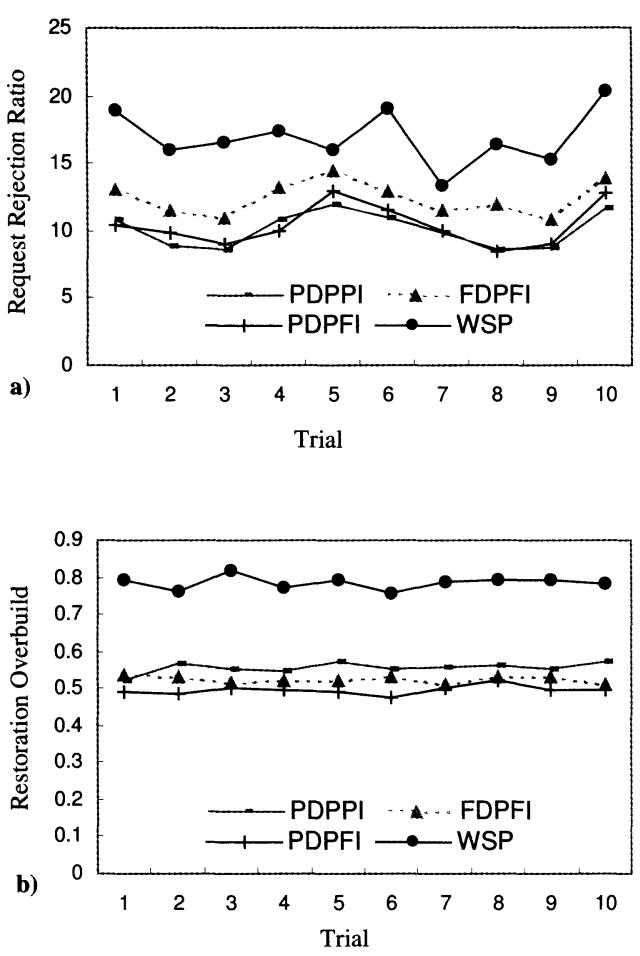

Figure 5 - a) Request rejection ratio b) Restoration overbuild.

number of links to protect at MPLS layer is less than the cases with 20 links and 24 links respectively (see Fig. 4b and $4 \mathrm{c}$ respectively), local protection is used more, decreasing the failure recovery time.

In Fig. 5.a, when the 20 percent of the links must be protected, the algorithms proposed in this paper (PDPPI, PDPFI) offer low request rejection ratio throughout the experiment. On the other hand, WSP algorithm presents worse request rejection ratio compared to the rest of algorithms since WSP does not take into account shared protection in its objectives.

Finally, in Fig. 5.b, the restoration overbuild is analyzed. In this case our proposals show a better behavior in terms of the bandwidth used than the rest of the algorithms.

Note that there is not a high improvement when Full Information Routing is used compared to the Partial Information Routing. Therefore, PDPPI can be a better choice than PDPFI since all the routing information is not always available, simplifying the management of the network.

\section{CONCLUSIONS}

In this paper a novel QoS with protection routing algorithm was introduced in a GMPLS-based network where MPLS requests were set up over an optical layer. The proposed algorithms took into account the multi-layer scenario in order to minimize the resource consumption. A partially protected optical layer was proposed and links that were protected at this layer were not again protected at
MPLS layer. Moreover, a new definition of link-disjoint path based on Shared Risk Link Group (SRLG) was made in order to share more backup bandwidth, minimizing the resource consumption. This paper took into consideration different levels of reliability and failure impact in terms of recovery time. Experiments showed the efficiency of the proposed methods in relation to previous ones, in terms of resources used to protect the network, the failure recovery time and the request rejection ratio.

\section{ACKNOWLEDGEMENTS}

This research was carried out at the Broadband and Wireless Networking Laboratory, Georgia Institute of Technology. Special thanks to Prof. Ian Akyildiz for facilitating my visit at his laboratory, to Dr. Caterina Scoglio for her constructive comments and to Dr. Apurva Mody for his support throughout the paper.

\section{REFERENCES}

[1] E. Mannie, "Generalized Multi-Protocol Label Switching architecture", RFC 3945, Oct. 2004.

[2] H. Zhang, A. Durresi, "Differentiated multi-layer survivability in IP/WDM networks", IEEE Network Operations and Management Symposium, Apr. 2002.

[3] K. Kar, M. Kodialam, T. V. Lakshman, "Routing restorable bandwidth guaranteed connections using maximum 2-route flows," IEEE/ACM Trans. on Networking, vol. 11, no. 5, Oct. 2003.

[4] P.-H. Ho et al. "On achieving optimal survivable routing for shared protection in survivable nextgeneration Internet," IEEE Trans. on Reliability, vol. 53, no. 2, pp. 216-225, Jun. 2004.

[5] E. Calle et al. "Enhancing fault management performance of two-step QoS routing algorithms in GMPLS," in Proc. of IEEE ICC, Jun. 2004.

[6] P. Sebos, J. Yates, G. Hjalmtysson, A. Greenberg, "Auto-discovery of Shared Risk Link Groups," in Proc. of the Optical Fiber Communication Conference and Exhibit (OFC), pp. WWD3-1-WWD3-3, March 2001.

[7] R. Bhandari, "Survivable networks: algorithms for diverse routing," Kluwer Academic Publishers, 1999.

[8] R. Guerin, D. Williams, A. Orda. "QoS routing mechanisms and OSPF extensions", in Proc. of IEEE Globecom 1997.

[9] E. Oki et al., "Dynamic multilayer routing schemes in GMPLS-based IP+Optical Networks," IEEE Communications Magazine, vol. 43, pp. 108-114, Jan. 2005.

[10] Murali Kodialam, T. V. Lakshman. "Dynamic routing of bandwidth guaranteed tunnels with restoration", in Proc. of IEEE Infocom 2000.

[11]G. Li et al. "Efficient distributed path selection for shared restoration connections", in Proc. of IEEE Infocom, 2002. 
[12] J. L. Marzo et al. "QoS on-line routing and MPLS multilevel protection: a survey," IEEE Communication Magazine, vol. 41, no. 10, pp. 126-132, October 2003.

[13] J. Tapolcai, P.-H. Ho, "Deeper study on segment shared protection," in Proc. of IEEE ISPAN, 2004.

[14]Q. Zheng, et al. "Protection approaches for dynamic traffic in IP/MPLS over WDM Networks," IEEE Optical Communications, vol. 41 , no. 5, pp. S24-S25, May 2003.
[15]A. Urra, E. Calle, J. L. Marzo, "Enhanced Protection using Shared Segment Backups in a Multiservice GMPLS-based Networks," in Proc. of IEEE ISCC, Jun. 2005. 